\title{
The acute impact of ingestion of breads of varying composition on blood glucose, insulin and incretins following first and second meals
}

\author{
Anita Mofidi Najjar ${ }^{1}$, Patricia M. Parsons ${ }^{1}$, Alison M. Duncan ${ }^{1}$, Lindsay E. Robinson ${ }^{1}$, Rickey Y. Yada ${ }^{2}$ \\ and Terry E. Graham ${ }^{1 *}$ \\ ${ }^{1}$ Department of Human Health and Nutritional Sciences, University of Guelph, Guelph, Ontario N1G 2W1, Canada \\ ${ }^{2}$ Department of Food Science, University of Guelph, Guelph, Ontario N1G 2W1, Canada
}

(Received 22 August 2007 - Revised 14 April 2008 - Accepted 28 April 2008 - First published online 23 June 2008)

Structural characteristics and baking conditions influence the metabolic responses to carbohydrate-containing foods. We hypothesized that consumption of whole wheat or sourdough breads would have a favourable effect on biomarkers of glucose homeostasis after first and second meals, compared with those for white bread. Ten overweight volunteers consumed $50 \mathrm{~g}$ available carbohydrate of each of the four breads (white, whole wheat, sourdough, whole wheat barley) followed $3 \mathrm{~h}$ later by a standard second meal. Blood was sampled for $3 \mathrm{~h}$ following bread ingestion and a further $2 \mathrm{~h}$ after the second meal for determination of glucose, insulin, paracetamol (indirect marker of gastric emptying), glucose-dependent insulinotropic polypeptide (GIP) and glucagon-like peptide-1 (GLP-1). Glucose and GLP-1 responses to sourdough bread were lower $(P<0.05)$ than whole wheat and whole wheat barley breads. Glucose area under the curve (AUC) for sourdough bread was lower than those for whole wheat $(P<0.005)$ and whole wheat barley $(P<0.03)$ breads for the entire study. GIP AUC after sourdough bread ingestion was lower compared to white $(P<0.004)$ and whole wheat barley $(P<0.002)$ breads following the second meal. There were no significant differences in insulin and paracetamol concentrations among the test breads. Ultra-fine grind whole wheat breads did not result in postprandial responses that were lower than those of white bread, but sourdough bread resulted in lower glucose and GLP-1 responses compared to those of these whole wheat breads following both meals.

Insulin sensitivity: Glucose-dependent insulinotropic polypeptide: Glucagon-like peptide-1: Whole wheat: Barley

North Americans spend at least $18 \mathrm{~h}$ a day in a postprandial state and postprandial responses to either carbohydrate $(\mathrm{CHO})$ or fat ingestion are associated with changes in various biomarkers for metabolic disorders such as type 2 diabetes and $\mathrm{CVD}^{(1,2)}$. Although $\mathrm{CHO}$ are a major component of the diet, the fundamental concept underlying the utility of glycaemic index and load is that minimizing the postprandial disturbances in blood glucose and insulin could have a beneficial effect on preventing and managing the metabolic disorders associated with type 2 diabetes.

Breads are a major component of the wide range of $\mathrm{CHO}$ containing foods in the North American diet ${ }^{(3,4)}$. The botanical origin and milling of the grains, processing of the dough and baking conditions can all influence the metabolic responses to the breads by influencing factors such as the rates of gastric emptying, hydrolysis of the starches and their absorption and hence the metabolic responses ${ }^{(5-8)}$. Strategies to optimize blood glucose and insulin responses to bread consumption include replacement of wheat flour with flour types richer in dietary fibres such as barley flour. Some studies $^{(9)}$ have suggested that $\beta$-glucan, the viscous fibre in barley, is capable of decreasing glucose and insulin responses by increasing the viscosity of the meal bolus, decreasing the rate of gastric emptying and consequently reducing the rate of intestinal absorption of $\mathrm{CHO}$ and glucose delivery to the blood. Another strategy to lower the metabolic response to bread is sourdough fermentation or the direct addition of organic acids. Organic acids are thought to prolong the rate of gastric emptying and/or decrease the rate of starch digestion but the mechanisms are not established ${ }^{(10-13)}$.

In recent years, investigations have suggested that the incretin hormones, glucose-dependent insulinotropic polypeptide (GIP) and glucagon-like peptide-1 (GLP-1) are intimately involved in the postprandial regulation of $\mathrm{CHO}$. These gutderived hormones have been shown to be responsible for approximately half of the postprandial insulin release and are thought to be important in pancreatic $\beta$ cell integrity. The major stimulus for incretin secretion is the presence of $\mathrm{CHO}$ in the gut ${ }^{(14-16)}$. However, our understanding of the impact of different types of $\mathrm{CHO}$ on the incretin response is in its infancy.

There is accumulating data suggesting that a low glycaemic index meal not only lowers the acute glycaemia but also improves glycaemia and insulinaemia after the

Abbreviations: AUC, area under the curve; CHO, carbohydrate; $C_{\max }$, peak serum concentration; GIP, glucose-dependent insulinotropic polypeptide; GLP-1, glucagon-like peptide-1; $T_{\max }$, time until peak serum concentration.

* Corresponding author: Dr Terry E. Graham, fax +1 519763 5902, email terrygra@uoguelph.ca 
subsequent (second) meal, a phenomenon called the second meal effect ${ }^{(17-20)}$. The physiological mechanism of how the glycaemia/insulinaemia of the first meal influences the second meal is not well-established. However, one theory is that the prolonged absorptive phase after the first meal will suppress SCFA release, thus improving insulin sensitivity at the time of the next meal ${ }^{(20)}$.

The primary objective of the present study was to determine the acute postprandial effect of $50 \mathrm{~g}$ available $\mathrm{CHO}$ in the form of breads made with different grain flours or leavening processes on the rate of gastric emptying and biomarkers of glucose homeostasis. A secondary objective was to examine the impact of these breads on the above biomarkers following consumption of a standard second meal. We hypothesized that white bread would be associated with the most extreme metabolic responses while more favourable responses would occur following ingestion of sourdough and whole wheat breads.

\section{Methods}

\section{Subjects}

Eleven overweight or obese males were recruited from the Guelph, Ontario vicinity through advertisement in local newspapers. The volunteers were all non-smokers and reported no history of gastrointestinal disease, gluten allergy, dyslipidaemia, diabetes or any other chronic diseases. They were not taking any medications (with the exception of two volunteers who were taking hypertension medication) or natural health products. Potential volunteers were screened for diabetes or glucose intolerance based on blood glucose at the end of a $2 \mathrm{~h}$ oral glucose tolerance test. The protocol of the study was approved by the University of Guelph Human Ethics Committee and written informed consent was obtained from all volunteers.

\section{Study protocol}

The volunteers were asked to maintain their usual diet and general lifestyle throughout the study, and to abstain from strenuous physical activity and ingestion of alcohol, caffeine substances and analgesic drugs that contain paracetamol for $48 \mathrm{~h}$ prior to testing. Dietary records were kept for the $3 \mathrm{~d}$ preceding each of the study days. The night before each study day, the volunteers were provided with the same commercially prepared frozen meal for consistency.

Each volunteer consumed four different test breads on separate occasions, at least 1 week apart, in a single blind, randomized, crossover design. On each occasion, the volunteer reported following a $12 \mathrm{~h}$, overnight fast. A venous catheter was inserted into the forearm by a trained technician, and kept clear for the duration of the experiment with a slow saline infusion. After a fasting blood sample was taken, volunteers consumed a serving of bread (without any spread) together with $250 \mathrm{ml}$ water within $10 \mathrm{~min}$. At $3 \mathrm{~h}$ after bread consumption, volunteers consumed a standard, commercially prepared lunch (6-inch Subway ${ }^{\circledR}$ sandwich on white bread with $300 \mathrm{ml}$ orange juice) within $15 \mathrm{~min}$ and were studied for an additional $2 \mathrm{~h}$. The serving of the test breads provided $50 \mathrm{~g}$ available $\mathrm{CHO}$ and $1 \mathrm{~g}$ paracetamol, required portions of $98,138,109$ and $127 \mathrm{~g}$ for white, whole wheat, sourdough and whole wheat barley bread, respectively.

Blood samples were taken at 0 (fasting), 15, 30, 60, 90, 120 and $180 \mathrm{~min}$ after the consumption of the bread as well as at $15,30,60,90$ and 120 min after consumption of the second meal.

\section{Study test breads}

Four different test breads (white bread, whole wheat bread, sourdough bread and whole wheat barley bread) were prepared for this study. Paracetamol $(13-14 \mathrm{~g})$ was added to the flour in order to estimate gastric emptying rate. The whole wheat and whole wheat barley breads had very similar formulations, differing only in the types of flour used. It should be noted that whole wheat and whole grain flours were ultra-fine ground flours due to the baking quality and had similar particle size. The white and sourdough breads also had similar recipes and the same white flour was used in the baking of both breads. The recipes are summarized in Table 1 . The primary investigator conducting the study prepared all breads in a commercial kitchen as follows: after mixing the ingredients, the fully developed dough relaxed at room temperature for $10 \mathrm{~min}$, then the dough was divided into $480 \mathrm{~g}$ pieces and proofed for $60 \mathrm{~min}$ at $30^{\circ} \mathrm{C}$ (relative humidity $70 \%$ ), except for the sourdough which was proofed at room temperature for $3 \mathrm{~h}$. Baking was performed at $180^{\circ} \mathrm{C}$ for $30 \mathrm{~min}$ and then the bread was allowed to cool at room temperature for about $4 \mathrm{~h}$. The crust was removed and three or four slices of bread were wrapped in aluminium foil and stored at $-20^{\circ} \mathrm{C}$ until used. The breads were heated in a microwave for $20 \mathrm{~s}$ before consumption.

A portion $(100 \mathrm{~g})$ of each study test bread was analysed by two different laboratories (Industrial Laboratories of Canada (ILC) in Tilsonburg, Ontario and Agriculture and Agri-Food Canada (AAFC) in Guelph, Ontario) and the results were averaged (Table 2).

\section{Biochemical and dietary analysis}

A single blood sample consisted of $14 \mathrm{ml}$ and was partitioned as follows: approximately $2 \mathrm{ml}$ blood for glucose analysis were put into tubes containing 72 United States Pharmacopeia units sodium heparin. Blood $(4 \mathrm{ml})$ for serum insulin and

Table 1. Study test bread recipes

\begin{tabular}{lcccc}
\hline & White & $\begin{array}{c}\text { Whole } \\
\text { wheat }\end{array}$ & Sourdough & $\begin{array}{c}\text { Whole wheat } \\
\text { barley }\end{array}$ \\
\hline White flour (g) & 1255 & & 1056 & \\
Whole wheat flour (g) & & 900 & & 922 \\
Whole grain flour (g) & & 365 & & 366 \\
Barley flour (g) & & & & 67 \\
Gluten (g) & & 39 & 633 & \\
Sourdough starter ${ }^{*}(\mathrm{~g})$ & 27 & 26 & 15 & 30 \\
Yeast (g) & 50 & 51 & 57 & 53 \\
Sucrose (g) & 25 & 26 & 28 & 27 \\
Salt (g) & 50 & 51 & 57 & 53 \\
Oil (g) & 13 & 13 & 13 & 14 \\
Paracetamol (g) & 761 & 912 & 470 & 949 \\
Water (g) & & & & \\
\hline
\end{tabular}

* Made from white flour, water and dry yeasts. 
Table 2. Nutrient composition of study test bread servings used to provide $50 \mathrm{~g}$ available carbohydrate $(\mathrm{CHO})^{*}$

\begin{tabular}{|c|c|c|c|c|}
\hline & White & $\begin{array}{l}\text { Whole } \\
\text { wheat }\end{array}$ & Sourdough & $\begin{array}{c}\text { Whole wheat } \\
\text { barley }\end{array}$ \\
\hline Portion size $(\mathrm{g})$ & 98 & 138 & 109 & 127 \\
\hline Available $\mathrm{CHO}(\mathrm{g}) \dagger$ & 50 & 50 & 50 & 50 \\
\hline Fat $(g)$ & $4 \cdot 2$ & $6 \cdot 1$ & $4 \cdot 7$ & $6 \cdot 1$ \\
\hline Protein (g) & $9 \cdot 7$ & $16 \cdot 0$ & $9 \cdot 8$ & $15 \cdot 1$ \\
\hline Total fibre $(\mathrm{g})$ & 1.5 & $6 \cdot 3$ & 1.0 & 5.5 \\
\hline Energy content (kJ) & $1182 \cdot 7$ & $1490 \cdot 3$ & $1194 \cdot 8$ & $1411 \cdot 6$ \\
\hline
\end{tabular}

*Test breads were analysed by two different laboratories and the results were averaged.

† Available $\mathrm{CHO}$ was calculated using the formula: total $\mathrm{CHO}$ - total dietary fibre.

paracetamol analysis was collected into tubes without any anticoagulants. After centrifugation $(1341 \mathrm{~g} ; 10 \mathrm{~min})$ at $4^{\circ} \mathrm{C}$, serum was stored at $-20^{\circ} \mathrm{C}$ for later analysis. For GIP total and GLP-1 total analysis, $6 \mathrm{ml}$ blood were collected into ice-chilled tubes containing $10.8 \mathrm{mg}$ potassium EDTA and $1824 \mathrm{kIU}$ aprotinin. Dipeptidylpeptidase-4 inhibitor $(10 \mu \mathrm{l} / \mathrm{ml}$ blood) was added to the samples in less than $30 \mathrm{~s}$. After centrifugation $(1000 \mathrm{~g} ; 15 \mathrm{~min})$ at $4^{\circ} \mathrm{C}$, plasma was stored at $-80^{\circ} \mathrm{C}$ until assayed.

All analyses were conducted in duplicate. Blood glucose was determined using a semiautomatic glucose and lactate analyser (STAT 2300; YSI, Yellow Springs, OH, USA). Serum insulin was analysed by RIA (Coat-A-Count, Diagnostic Product Corporation, Los Angeles, USA). The calculated inter-assay CV for serum insulin was $2.6 \%$. Serum paracetamol concentration was determined using a paracetamol assay kit (Cambridge Life Science, UK). The inter-assay CV for serum paracetamol was $3.7 \%$. The plasma GIP total concentrations were determined by GIP total ELISA kit (Linco Research Inc., St. Charles, MO, USA). The inter-assay CV for plasma GIP total was $6 \cdot 6 \%$. The plasma GLP-1 total concentrations were measured by GLP-1 total RIA kit (Linco Research Inc.) after extraction with $70 \%$ ethanol. The inter-assay CV for plasma GLP-1 total was $7 \cdot 2 \%$. For all of the assays, all samples from each subject were analysed together to eliminate the effect of inter-batch variation.

Energy and nutrient intakes were calculated by using the ESHA Food Processor program (version 9.5; Salem, OR, USA). The data were analysed for energy, macronutrients, cholesterol and dietary fibre. Intake data were averaged across the $3 \mathrm{~d}$.

\section{Calculations and statistical analysis}

Area under the curve (AUC) was determined for blood glucose, serum insulin and paracetamol, plasma GIP and GLP-1 (GraphPad Prism, version 3.0; San Diego, CA, USA). Data were analysed for the time periods $0-300 \mathrm{~min}$ (defined as entire study), $0-180$ min (defined as the first meal) and $180-300 \mathrm{~min}$ (defined as the second meal). Fasting $(0 \mathrm{~min})$ concentrations were used as baseline in second meal AUC calculations. Insulin sensitivity index was calculated using the method described by Matsuda \& DeFronzo ${ }^{(21)}$. For the characterization of paracetamol absorption, the ratio of peak serum concentration $\left(C_{\max }, \mu \mathrm{mol} / \mathrm{l}\right)$ to time until peak concentration $\left(T_{\max }, \min \right)$ was calculated ${ }^{(22)}$.
All statistical analyses were performed using the Statistical Analysis System (SAS Institute Inc., version 9.1; Cary, NC, USA). Significance $(P<0 \cdot 05)$ was tested by two-factor repeated measure ANOVA using a mixed model (treatment: fixed effect and volunteer: random effect) followed by the Tukey's test for multiple comparisons. Results are given as means and their standard errors.

\section{Results}

A total of eleven volunteers were recruited for the study. One volunteer discontinued because of illness. Based on this, the statistical analysis was performed on a total of ten volunteers (age 59.0 (SE 2.41) years; BMI $30 \cdot 8($ SE 0.95$) \mathrm{kg} / \mathrm{m}^{2}$ ).

\section{Test breads}

The $\mathrm{pH}$ of both white and sourdough breads were measured and sourdough had lower $\mathrm{pH}$ compared to the white bread (5.07 v. 5.77, respectively) which is indicative of the increased level of organic acids in sourdough bread.

\section{Blood glucose}

The mean blood glucose response to the test breads following consumption of the first and second meals is shown in Fig. 1. Fasting blood glucose concentrations did not significantly differ among the test breads. There were significant differences in overall glucose responses to the test breads with sourdough being lower than both white $(P<0.05)$ and whole wheat $(P<0.007)$ breads as well as whole wheat barley being lower than whole wheat $(P<0.05)$ bread. AUC of the glucose response to the test breads is displayed in Table 3. For each meal period as well as entire study, glucose AUC for sourdough was lower than whole wheat $(P<0.05)$ and for both the second meal period and the entire study sourdough was also lower than whole wheat barley $(P<0.05)$ bread.

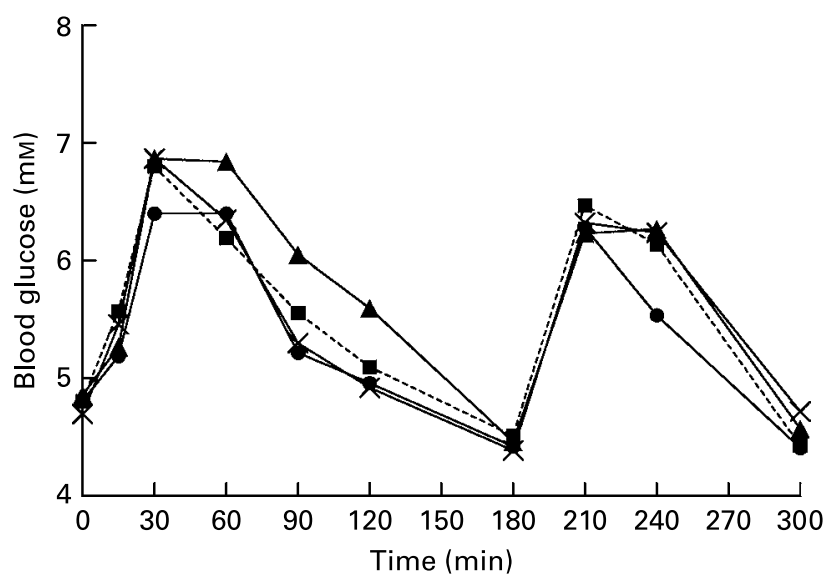

Fig. 1. Mean fasting and postprandial glucose responses to the test breads $(\mathbf{\square}$, white; $\boldsymbol{\Lambda}$, whole wheat; $\boldsymbol{\bullet}$, sourdough; $\times$, whole wheat barley) after the first and second meals. Test bread was ingested at $0 \mathrm{~min}$ followed by ingestion of a standardized lunch meal at $180 \mathrm{~min}$. Significant overall treatment effects were found (sourdough was lower than both white and whole wheat breads, whole wheat was higher than whole wheat barley bread). Values are means and standard errors are not included for the clarity of the figure $(n 10)$. 
Table 3. Area under the curve of blood glucose and serum insulin responses to the test breads for the entire study, first meal and second meal ${ }^{\star}$ (Mean values with their standard errors for ten subjects)

\begin{tabular}{|c|c|c|c|c|c|c|c|c|}
\hline \multirow[b]{2}{*}{ Time/parameter } & \multicolumn{2}{|c|}{ White } & \multicolumn{2}{|c|}{ Whole wheat } & \multicolumn{2}{|c|}{ Sourdough } & \multicolumn{2}{|c|}{ Whole wheat barley } \\
\hline & Mean & SEM & Mean & SEM & Mean & SEM & Mean & SEM \\
\hline Glucose (mmol/l; $5 \mathrm{~h})$ & $222 \cdot 1^{a, b}$ & $40 \cdot 7$ & $272 \cdot 7^{c}$ & $52 \cdot 4$ & $161 \cdot 8^{\mathrm{b}}$ & $39 \cdot 3$ & $250 \cdot 5^{\mathrm{a}, \mathrm{c}}$ & $37 \cdot 3$ \\
\hline Insulin (nmol/l; 5h) & $66 \cdot 9^{\mathrm{a}}$ & $25 \cdot 3$ & $66 \cdot 3^{a}$ & $25 \cdot 2$ & $63 \cdot 3^{a}$ & $24 \cdot 3$ & $57 \cdot 4^{\mathrm{a}}$ & $21 \cdot 7$ \\
\hline \multicolumn{9}{|l|}{ First meal (0-180 min) } \\
\hline Glucose (mmol/l; $3 \mathrm{~h})$ & $124 \cdot 0^{a, b}$ & 28.5 & $167 \cdot 7^{\mathrm{a}}$ & $32 \cdot 9$ & $96 \cdot 3^{b}$ & $31 \cdot 3$ & $131 \cdot 7^{a, b}$ & $21 \cdot 0$ \\
\hline Insulin (nmol/l; 3h) & $39 \cdot 6^{\mathrm{a}}$ & $15 \cdot 0$ & $39 \cdot 2^{\mathrm{a}}$ & $15 \cdot 0$ & $37 \cdot 5^{\mathrm{a}}$ & $14 \cdot 2$ & $33 \cdot 9^{\mathrm{a}}$ & $12 \cdot 8$ \\
\hline \multicolumn{9}{|c|}{ Second meal (180-300 min) } \\
\hline Glucose (mmol/l; 2h) & $98 \cdot 1^{a, b}$ & $12 \cdot 2$ & $105 \cdot 0^{\mathrm{a}}$ & $19 \cdot 5$ & $65 \cdot 5^{b}$ & $8 \cdot 1$ & $118 \cdot 8^{a}$ & $16 \cdot 3$ \\
\hline Insulin (nmol/l; 2 h) & $27 \cdot 3^{a}$ & $10 \cdot 3$ & $27 \cdot 1^{\mathrm{a}}$ & $10 \cdot 2$ & $25 \cdot 8^{a}$ & $9 \cdot 8$ & $23 \cdot 3^{a}$ & 8.9 \\
\hline
\end{tabular}

a,b,c Mean values within a row with unlike superscript letters were significantly different $(P<0.05)$.

${ }^{\star}$ For details of subjects and procedures, see Methods.

\section{Serum insulin and insulin sensitivity}

Fasting serum insulin concentrations were not significantly different among the test breads despite the differences in glucose. There were no significant differences in overall insulin responses to the test breads (Fig. 2). Similarly, no significant difference was found in insulin AUC among the test breads (Table 3). In addition, there were no significant differences in insulin sensitivity index among the test breads (data not shown).

Plasma glucose-dependent insulinotropic polypeptide and glucagon-like peptide-1

Fasting GIP and GLP-1 concentrations did not significantly differ among the test breads. No overall treatment effect was found in GIP responses to the test breads (Fig. 3). While there were no significant differences for the GIP AUC among the test breads following the first meal, the GIP AUC after sourdough bread ingestion was less than white

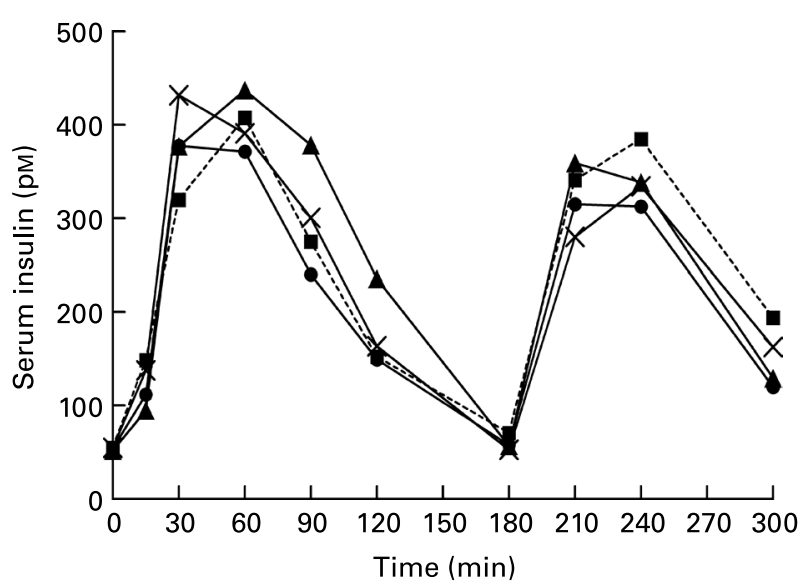

Fig. 2. Mean fasting and postprandial serum insulin responses to the test breads ( $\mathbf{\square}$, white; $\boldsymbol{\Lambda}$, whole wheat; $\bullet$, sourdough; $\times$, whole wheat barley) after the first and second meals. Test bread was ingested at 0 min followed by ingestion of a standardized lunch meal at $180 \mathrm{~min}$. Significant overall treatment effects were not found. Values are means and standard errors are not included for the clarity of the figure $(n 10)$.
$(P<0.004)$ and whole wheat barley $(P<0.002)$ breads after the second meal (Table 4). Postprandial GLP-1 concentrations following ingestion of the test breads did not follow a pattern similar to that of GIP concentrations. There were significant differences in the overall GLP-1 responses to the test breads (Fig. 4) with the GLP-1 response to sourdough being lower than white $(P<0.0001)$ and whole wheat $(P<0.0001)$ breads. In addition, the GLP-1 concentration for white bread was lower than whole wheat barley bread $(P<0.02)$. The overall GLP-1 response to whole wheat barley was lower than whole wheat bread $(P<0.03)$. Despite the significant treatment effect in overall GLP-1 response, no significant difference was found in GLP-1 AUC among the test breads (Table 4).

\section{Serum paracetamol}

There was no significant difference in paracetamol concentrations at any time-points among the breads (Fig. 5). Furthermore, no difference was found in paracetamol AUC and

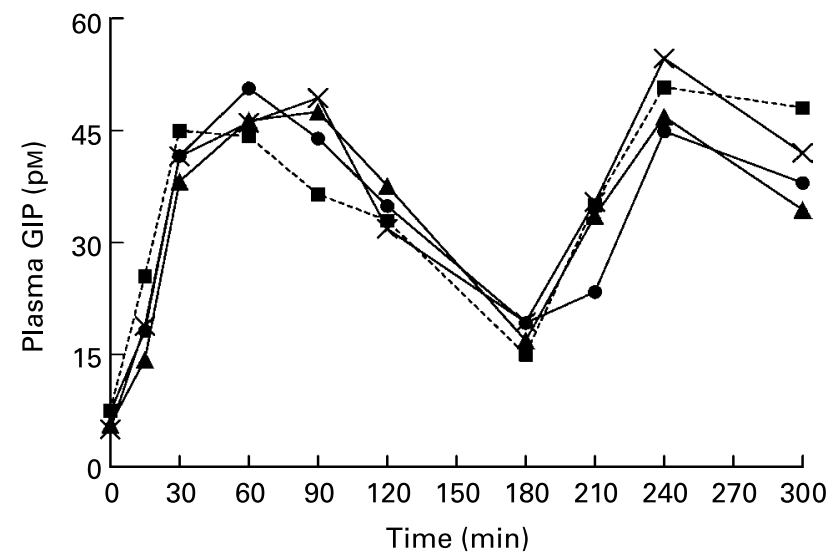

Fig. 3. Mean fasting and postprandial glucose-dependent insulinotropic polypeptide (GIP) responses to the test breads ( $\mathbf{\square}$, white; $\boldsymbol{\Lambda}$, whole wheat; $\boldsymbol{\bullet}$, sourdough; $\times$, whole wheat barley) after the first and second meals. Test bread was ingested at 0 min followed by ingestion of a standardized lunch meal at $180 \mathrm{~min}$. Significant overall treatment effects were not found. Values are means and standard errors are not included for the clarity of the figure $(n 10)$. 
Table 4. Area under the curve of plasma glucose-dependent insulinotropic polypeptide (GIP) and glucagon-like peptide-1 (GLP-1) responses to the test breads for the entire study, first meal and second meal ${ }^{*}$

(Mean values with their standard errors for ten subjects)

\begin{tabular}{|c|c|c|c|c|c|c|c|c|}
\hline \multirow[b]{2}{*}{ Time/parameter } & \multicolumn{2}{|c|}{ White } & \multicolumn{2}{|c|}{ Whole wheat } & \multicolumn{2}{|c|}{ Sourdough } & \multicolumn{2}{|c|}{ Whole wheat barley } \\
\hline & Mean & SEM & Mean & SEM & Mean & SEM & Mean & SEM \\
\hline \multicolumn{9}{|c|}{ Entire study (0-300 min) } \\
\hline GIP (nmol/l; $5 \mathrm{~h})$ & $8 \cdot 8^{a}$ & 0.8 & $8 \cdot 9^{a}$ & $0 \cdot 8$ & $8 \cdot 2^{a}$ & 0.9 & $9 \cdot 7^{\mathrm{a}}$ & $1 \cdot 2$ \\
\hline GLP-1 (nmol/l; 5h) & $1 \cdot 7^{\mathrm{a}}$ & 0.5 & $1.5^{\mathrm{a}}$ & 0.5 & $1 \cdot 0^{\mathrm{a}}$ & 0.4 & $1 \cdot 1^{\mathrm{a}}$ & 0.4 \\
\hline \multicolumn{9}{|l|}{ First meal (0-180 min) } \\
\hline GIP (nmol/l; 3h) & $4 \cdot 4^{\mathrm{a}}$ & 0.5 & $5 \cdot 1^{\mathrm{a}}$ & 0.4 & $4 \cdot 9^{\mathrm{a}}$ & 0.6 & $5 \cdot 2^{\mathrm{a}}$ & 0.7 \\
\hline \multicolumn{9}{|c|}{ Second meal (180-300 min) } \\
\hline GIP (nmol/l; 2 h) & $4 \cdot 4^{\mathrm{a}, \mathrm{c}}$ & 0.4 & $3 \cdot 8^{\mathrm{b}, \mathrm{c}}$ & 0.4 & $3 \cdot 3^{b}$ & 0.3 & $4 \cdot 5^{\mathrm{a}, \mathrm{c}}$ & 0.5 \\
\hline GLP-1 (nmol/l; $2 \mathrm{~h})$ & $0.9^{a}$ & 0.2 & $0.5^{\mathrm{a}}$ & 0.1 & $0.5^{\mathrm{a}}$ & 0.2 & $0.5^{\mathrm{a}}$ & 0.1 \\
\hline
\end{tabular}

a,b,c Mean values within a row with unlike superscript letters were significantly different $(P<0.05)$.

${ }^{*}$ For details of subjects and procedures, see Methods.

$C_{\max }: T_{\max }$ among the breads (data not shown), suggesting that gastric emptying was not different among the treatments.

\section{Discussion}

We examined the postprandial and second meal effects of ingestion of various breads in sedentary, overweight and obese men as this population represents a group that are at increased risk for type 2 diabetes. The breads were designed to cover a broad range of properties that have been suggested to influence $\mathrm{CHO}$ assimilation. The nature of the breads ingested influenced the metabolic responses to not only their own assimilation but also to the processing of a standard second meal $3 \mathrm{~h}$ later. However, the results from the present study do not support our hypothesis that white bread would induce the highest postprandial responses in blood glucose, insulin and incretins following both the first and second

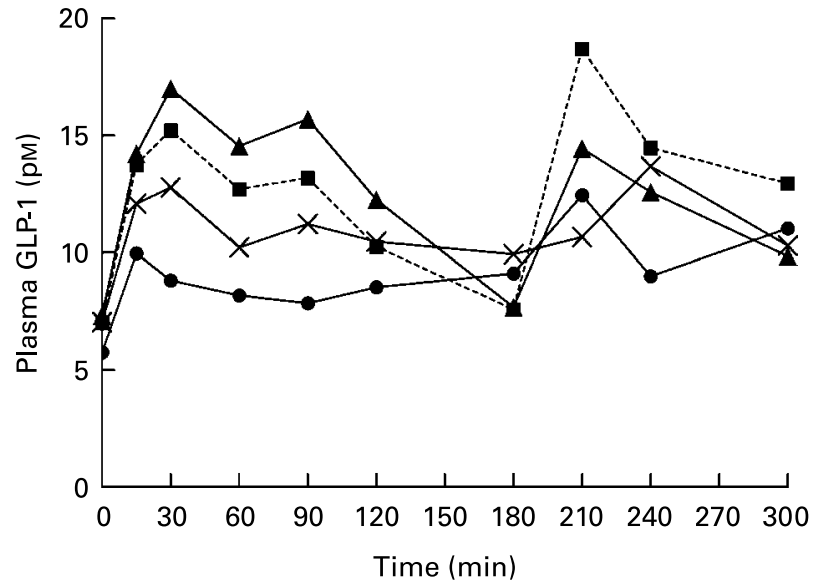

Fig. 4. Mean fasting and postprandial glucagon-like peptide-1 (GLP-1) responses to the test breads $(\boldsymbol{\square}$, white; $\boldsymbol{\Lambda}$, whole wheat; $\bullet$, sourdough; $\times$, whole wheat barley) after the first and second meals. Test bread was ingested at 0 min followed by ingestion of a standardized lunch meal at $180 \mathrm{~min}$. A significant overall treatment effect was identified (sourdough was lower than white and whole wheat breads and white was greater than whole wheat barley bread, whole wheat was greater than whole wheat barley bread). Values are means and standard errors are not included for the clarity of the figure $(n 10)$. meals. For most measures, ingestion of sourdough bread induced the least disturbance in $\mathrm{CHO}$ homeostasis while ultra-fine grind whole wheat bread generally induced the highest postprandial glucose responses in the volunteers.

The impact of sourdough bread ingestion on blood glucose responses was internally consistent with other parameters measured in the current study. The overall glucose response to the sourdough bread was significantly lower than white bread $(P<0 \cdot 05)$. Glucose AUC for sourdough bread was significantly lower than for whole wheat $(P<0.005)$ and whole wheat barley $(P<0.03)$ breads for the entire study. The more modest blood glucose disturbance together with no differences between breads for serum insulin, suggests that sourdough may have increased insulin sensitivity in these individuals. While the differences were not significant, the insulin sensitivity index after the ingestion of sourdough bread was 20-25\% higher than after whole wheat bread consumption in the first and second meals (data not shown).

The present findings agree with those of a previous study in which lowered glycaemia was noted with a sourdough bread containing lactic acid compared to a whole wheat bread ${ }^{(12)}$.

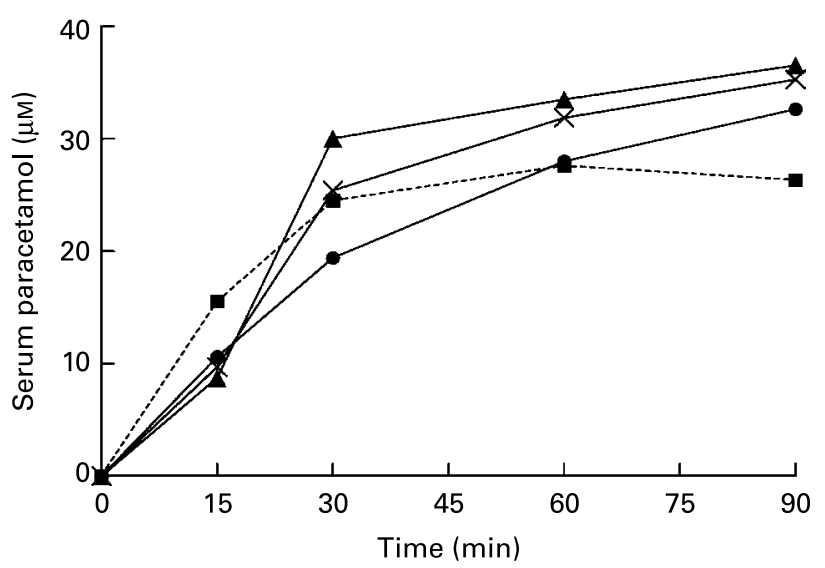

Fig. 5. Mean fasting and postprandial paracetamol responses to the test breads $(\boldsymbol{\square}$, white; $\boldsymbol{\Lambda}$, whole wheat; $\bullet$, sourdough; $\times$, whole wheat barley) over $90 \mathrm{~min}$ after the first meal. Test bread was ingested at $0 \mathrm{~min}$. There were no significant differences in paracetamol concentrations at any timepoint among the test breads. Values are means and standard errors are not included for the clarity of the figure $(n 10)$. 
Improved glycaemia following a bread meal supplemented with vinegar (acetic acid) has also been reported ${ }^{(23)}$. In the present study, lowered $\mathrm{pH}$ of sourdough bread compared with white bread is indicative of the increased level of organic acids in sourdough bread. It has been suggested that the presence of organic acids could reduce the gastric emptying rate ${ }^{(10,23)}$. In the present study no significant differences in postprandial paracetamol concentrations were noted (AUC and $T_{\max }: C_{\max }$ were similar), indicating that the rate of gastric emptying was not different among the test breads. This implies that the presence of organic acids in the sourdough bread interfered with the rate of digestion. Östman et al. ${ }^{(24)}$ reported that lactic acid either produced during sourdough fermentation or added directly to the bread reduced the rate of in vitro starch hydrolysis, suggesting that lactic acid interferes with the digestive process. It is possible that lactic acid in sourdough bread causes interaction between the gluten and starch, resulting in reduced starch bioavailability ${ }^{(24)}$. More work is needed to clarify the mechanism by which organic acids present in sourdough bread improve glycaemia.

The observed metabolic effects were carried forward to the next meal as the glucose AUC in the second meal period of the sourdough trial was reduced significantly compared to the whole wheat $(P<0 \cdot 03)$ and whole wheat barley $(P<0.005)$ trials. Östman et al. ${ }^{(13)}$ found that the addition of lactic acid to barley bread reduced the glycaemic and insulinaemic responses to a second meal (a high glycaemic index lunch) by $25 \%$ in healthy individuals. They speculated that this was due to the prolonged digestive phase after the first meal suppressing the plasma levels of SCFA and that this improved insulin sensitivity at the time of the next meal. The incretin results in the present study may also offer an explanation, as discussed later.

Recently there has been a great deal of attention to the responses of the incretins, GIP and GLP-1, in the ingestion of $\mathrm{CHO}$ and their impact on postprandial metabolism. Little is known about the effect of different types of $\mathrm{CHO}$ on secretion of incretins. In the current study, ingestion of sourdough bread resulted in a lower overall GLP-1 response compared with any of the other breads. In addition, the GIP AUC in the second meal was lower by 33 and $36 \%$ following ingestion of sourdough bread compared to white $(P<0.004)$ and whole wheat barley $(P<0.002)$ breads, respectively. Up to $50 \%$ of insulin secretion in response to feeding has been attributed to the incretins ${ }^{(14,15)}$. However, in the present study the nature of the breads was associated with marked differences in the incretin and blood glucose responses but not that for insulin. This implies that the relationship between the incretins-insulin-glucose is not straightforward in these volunteers. GLP-1 has also been proposed to enhance insulin sensitivity $^{(25)}$ and in the present investigation, sourdough had the lowest GLP-1 responses, no impact on insulin and yet there was a reduced AUC for glucose.

Similarly, Juntunen et al. ${ }^{(26)}$ found that ingestion of white bread, in comparison to rye bread (with or without the addition of $\beta$-glucan), did not affect blood glucose responses or rates of gastric emptying. However, while the insulin, GIP and GLP-1 responses to the two rye breads were significantly different from the responses to the white breads, there was not an obvious, direct relationship among the parameters. In addition, Bakhøj et al. ${ }^{(27)}$ showed that ingestion of Einkorn honey-salt leavened and whole grain breads reduced the postprandial GIP, but not that of GLP-1, compared to the conventional yeast bread and proposed that this was due to an increased level of organic acids (based on reduction of the $\mathrm{pH}$ in the dough). These studies as well as the present investigation demonstrate that the nature of bread results in altered blood glucose and/or insulin responses as well as those of the incretins. However, the mechanisms are complex and the physiological significance remains to be elucidated.

Dietary recommendations have emphasized the replacement of white bread with whole wheat bread or dark bread to improve glycaemia in individuals at risk for type 2 diabetes. However, in the present study, the ultra-fine grind whole wheat bread generally resulted in higher glycaemic responses compared to white bread. This finding may be attributed to the small particle size of the ultra-fine ground whole wheat flour used in the present study and the results should not be generalized to all whole wheat breads. Furthermore, it has been reported that only soluble fibre has the ability to lower postprandial glucose and insulin responses whereas insoluble fibre has negligible effects on glycaemia ${ }^{(28)}$. Soluble dietary fibre increases the viscosity and decreases gastrointestinal motility and subsequently reduces the rate of delivery of glucose to the blood ${ }^{(9,29,30)}$. While we only determined total fibre, it is possible that the total fibre content of our whole wheat bread (without barley) consisted predominantly of insoluble fibre.

The most surprising finding of the present study was that the whole wheat breads resulted in the greatest disturbances in $\mathrm{CHO}$ homeostasis. This finding should not be interpreted as reflecting that whole wheat products are unhealthy. The present study only examined breads made from one type of ultra-fine grind flour and only examined $\mathrm{CHO}$ homeostasis. As noted earlier, the nature of the flour selected for the whole wheat bread is a limitation, as is our small study sample. We employed a number of pre-test controls to reduce the intra-subject variability, including restricting exercise and providing a standardized dinner to be consumed the night before each study day. The baseline data had very little variation, suggesting that these controls were effective. We also reduced the contribution of inter-individual variation by using a randomized crossover study design such that each subject was their own control. Nevertheless, we acknowledge that there could be a type 2 statistical error masking potential biological effects that we could not detect. However, it is noteworthy that the data are internally very consistent. For example, sourdough bread had the lowest responses in virtually every measure.

An important limitation of this investigation is that in order to equalize the amount of available $\mathrm{CHO}(50 \mathrm{~g})$ across treatments, the whole wheat and whole wheat barley challenges resulted in the volunteers ingesting 30-40\% more mass (in comparison to white bread) as well as greater amounts of energy, fat, protein and fibre content. While the difference in other nutrient components could influence the results, these would have been expected to slow gastric emptying rate, delay $\mathrm{CHO}$ absorption and lessen the rise in blood glucose in whole wheat bread trials. While factors such as energy, fat, protein and fibre consumed can be important, in the present study they could not account for the differences observed between sourdough and the whole wheat breads. It may be that any putative positive effects on glycaemia are only 
apparent after long-term dietary interventions. Further work is thus necessary to elucidate if these factors impact the metabolic responses by altering the digestion and absorption of the bread consumed.

\section{Conclusion}

The ingestion of $50 \mathrm{~g}$ available $\mathrm{CHO}$ in the form of different breads by middle-aged overweight or obese men influenced not only the postprandial response to this meal but also those of a standardized second meal. Contrary to our hypothesis, the ingestion of white bread did not result in postprandial glycaemic or insulinaemic responses that were greater than those of ultra-fine grind whole wheat breads. In addition, the consumption of sourdough bread resulted in lower overall glucose and GLP-1 responses compared to those of these whole wheat and whole wheat barley breads.

\section{Acknowledgements}

We wish to thank Hayhoe Mills Ltd and ADM Milling Company for providing the flour for the breads and the Food Development Group (Dr Phillip Lee Wing) for assisting with developing the bread recipes. Special thanks to Premila Sathasivam and Mehrnoosh Kashani for their excellent technical assistance. The financial support from the Food Research Program of the Ontario Ministry of Agriculture and Food and Rural Affairs is greatly appreciated. This work was also supported by an industrial NSERC scholarship for A. M. N. sponsored by Stone Millhouse Bakery. A. M. N. assisted with the protocol development, recruited the subjects, co-ordinated the study, collected and interpreted the data, and drafted the manuscript. P. M. P. assisted with protocol development and study co-ordination. A. M. D. contributed to the study design, protocol development, statistical analysis and manuscript revision. L. E. R. assisted with the protocol development and manuscript revision. R. Y. Y. assisted with the protocol development. T. E. G. designed the study, supervised the research, directed the data analysis and interpretation, and provided significant assistance during manuscript preparation. None of the authors have conflicts of interest.

\section{References}

1. Abbott WG, Howard BV, Christin L, Freymond D, Lillioja S, Boyce VL, Anderson TE, Bogardus C \& Ravussin E (1988) Short-term energy balance: relationship with protein, carbohydrate, and fat balances. Am J Physiol 255, E332-E337.

2. Graham TE \& Adamo KB (1999) Dietary carbohydrate and its effects on metabolism and substrate stores in sedentary and active individuals. Can J Appl Physiol 24, 393-415.

3. Gray-Donald K, Jacobs-Starkey L \& Johnson-Down L (2000) Food habits of Canadians: reduction in fat intake over a generation. Can J Public Health 91, 381-385.

4. Phillips S, Jacobs SL \& Gray-Donald K (2004) Food habits of Canadians: food sources of nutrients for the adolescent sample. Can J Diet Pract Res 65, 81-84.

5. Hoebler C, Karinthi A, Chiron H, Champ M \& Barry JL (1999) Bioavailability of starch in bread rich in amylose: metabolic responses in healthy subjects and starch structure. Eur J Clin Nutr 53, 360-366.
6. Holm J \& Bjorck I (1992) Bioavailability of starch in various wheat-based bread products: evaluation of metabolic responses in healthy subjects and rate and extent of in vitro starch digestion. Am J Clin Nutr 55, 420-429.

7. Liljeberg H, Granfeldt Y \& Bjorck I (1992) Metabolic responses to starch in bread containing intact kernels versus milled flour. Eur J Clin Nutr 46, 561-575.

8. Snow P \& O'Dea K (1981) Factors affecting the rate of hydrolysis of starch in food. Am J Clin Nutr 34, 2721-2727.

9. Wursch P \& Pi-Sunyer FX (1997) The role of viscous soluble fiber in the metabolic control of diabetes. A review with special emphasis on cereals rich in beta-glucan. Diabetes Care 20, $1774-1780$

10. Liljeberg H \& Bjorck I (1998) Delayed gastric emptying rate may explain improved glycaemia in healthy subjects to a starchy meal with added vinegar. Eur J Clin Nutr 52, 368-371.

11. Liljeberg HG, Lonner CH \& Bjorck IM (1995) Sourdough fermentation or addition of organic acids or corresponding salts to bread improves nutritional properties of starch in healthy humans. J Nutr 125, 1503-1511.

12. Liljeberg HG \& Bjorck IM (1996) Delayed gastric emptying rate as a potential mechanism for lowered glycemia after eating sourdough bread: studies in humans and rats using test products with added organic acids or an organic salt. Am J Clin Nutr 64, 886-893.

13. Ostman EM, Liljeberg Elmstahl HG \& Bjorck IM (2002) Barley bread containing lactic acid improves glucose tolerance at a subsequent meal in healthy men and women. J Nutr 132, $1173-1175$.

14. Burcelin $\mathrm{R}$ (2005) The incretins: a link between nutrients and well-being. Br J Nutr 93, Suppl. 1, S147-S156.

15. Drucker DJ (2006) The biology of incretin hormones. Cell Metab 3, 153-165.

16. Holst JJ \& Gromada J (2004) Role of incretin hormones in the regulation of insulin secretion in diabetic and nondiabetic humans. Am J Physiol Endocrinol Metab 287, E199-E206.

17. Axelsen M, Arvidsson LR, Lonnroth P \& Smith U (1999) Breakfast glycaemic response in patients with type 2 diabetes: effects of bedtime dietary carbohydrates. Eur J Clin Nutr 53, $706-710$.

18. Clark CA, Gardiner J, McBurney MI, Anderson S, Weatherspoon LJ, Henry DN \& Hord NG (2006) Effects of breakfast meal composition on second meal metabolic responses in adults with type 2 diabetes mellitus. Eur J Clin Nutr 60, $1122-1129$.

19. Liljeberg HG, Akerberg AK \& Bjorck IM (1999) Effect of the glycemic index and content of indigestible carbohydrates of cereal-based breakfast meals on glucose tolerance at lunch in healthy subjects. Am J Clin Nutr 69, 647-655.

20. Wolever TM, Jenkins DJ, Ocana AM, Rao VA \& Collier GR (1988) Second-meal effect: low-glycemic-index foods eaten at dinner improve subsequent breakfast glycemic response. Am J Clin Nutr 48, 1041-1047.

21. Matsuda M \& DeFronzo RA (1999) Insulin sensitivity indices obtained from oral glucose tolerance testing: comparison with the euglycemic insulin clamp. Diabetes Care 22, 1462-1470.

22. Willems M, Quartero AO \& Numans ME (2001) How useful is paracetamol absorption as a marker of gastric emptying? A systematic literature study. Dig Dis Sci 46, 2256-2262.

23. Ostman E, Granfeldt Y, Persson L \& Bjorck I (2005) Vinegar supplementation lowers glucose and insulin responses and increases satiety after a bread meal in healthy subjects. Eur $J$ Clin Nutr 59, 983-988.

24. Ostman EM, Nilssont M, Liljeberg-Elmstahl HG, Molin G \& Bjorck I (2002) The effect of lactic acid on blood glucose and insulin responses to cereal products: mechanistic studies in healthy subjects and in vitro. J Cereal Sci 36, 339-346. 
25. Egan JM, Meneilly GS, Habener JF \& Elahi D (2002) Glucagon-like peptide-1 augments insulin-mediated glucose uptake in the obese state. J Clin Endocrinol Metab 87, 3768-3773.

26. Juntunen KS, Niskanen LK, Liukkonen KH, Poutanen KS, Holst JJ \& Mykkanen HM (2002) Postprandial glucose, insulin, and incretin responses to grain products in healthy subjects. Am J Clin Nutr 75, 254-262.

27. Bakhøj S, Flint A, Holst JJ \& Tetens I (2003) Lower glucosedependent insulinotropic polypeptide (GIP) response but similar glucagon-like peptide 1 (GLP-1), glycaemic, and insulinaemic response to ancient wheat compared to modern wheat depends on processing. Eur J Clin Nutr 57, 1254-1261.

28. Tabatabai A \& Li S (2000) Dietary fiber and type 2 diabetes. Clin Excell Nurse Pract 4, 272-276.

29. Jenkins DJ, Jenkins AL, Wolever TM, Rao AV \& Thompson LU (1986) Fiber and starchy foods: gut function and implications in disease. Am J Gastroenterol 81, 920-930.

30. Jenkins DJ, Axelsen M, Kendall CW, Augustin LS, Vuksan V \& Smith U (2000) Dietary fibre, lente carbohydrates and the insulin-resistant diseases. Br J Nutr 83, Suppl. 1, S157-S163. 